\title{
Cervico-Isthmic Pregnancy on Cesarean Scar Successful Treated with Methotrexate, Mifepristone and Misoprostol: A Case Report
}

\author{
K. E. Logbo-Akey ${ }^{*}$, D. R. Ajavon², K. B. M'bortche ${ }^{3}$, P. Y. Kambote ${ }^{1}$, A. S. Aboubakari ${ }^{1}$, K. Akpadza ${ }^{4}$ \\ ${ }^{1}$ Gynecology and Obstetrics Department of Kara University Hospital, University of Kara, Kara, Togo \\ ${ }^{2}$ Gynecology and Obstetrics Department of CHR Tomdé, University of Kara, Kara, Togo \\ ${ }^{3}$ Togolese Association of Family Well-Being Planning Center (ATBEF), University of Lomé, Lomé, Togo \\ ${ }^{4}$ Gynecology and Obstetrics Department of Sylvanius Olympio University Hospital, University of Lomé, Lomé, Togo \\ Email: *edemattis@yahoo.fr
}

How to cite this paper: Logbo-Akey, K.E., Ajavon, D.R., M’bortche, K.B., Kambote, P.Y., Aboubakari, A.S. and Akpadza, K. (2021) Cervico-Isthmic Pregnancy on Cesarean Scar Successful Treated with Methotrexate, Mifepristone and Misoprostol: A Case Report. Open Journal of Obstetrics and Gynecology, 11, 1229-1235.

https://doi.org/10.4236/ojog.2021.119116

Received: August 24, 2021

Accepted: September 24, 2021

Published: September 27, 2021

Copyright $\odot 2021$ by author(s) and Scientific Research Publishing Inc. This work is licensed under the Creative Commons Attribution International License (CC BY 4.0).

http://creativecommons.org/licenses/by/4.0/

\begin{abstract}
Introduction: Cervico-isthmic pregnancy is rare, and serious because of its hemorrhagic complication. Its management varies according to the teams. Observation: We report a case of cervico-isthmic pregnancy on a cesarean scar. This is a 35-year-old, G2P2, who consulted for bleeding from the 1st trimester of pregnancy. The clinical examination found a minimal uterine bleeding. The diagnosis was made by a vaginal ultrasound which found a cervico-isthmic implantation of the pregnancy on the caesarean scar. A protocol of Mifepristone and Misoprostol followed by administration of a single dose of $1 \mathrm{mg} / \mathrm{kg}$ of Methotrexate was performed. Cure was obtained 1 month after treatment by negativation of plasma HCG. No bleeding complications were noted. A follow-up ultrasound performed 2 months later showed a uterine vacuity and the presence of an isthmocoele. It was in fact a pregnancy that had implanted in the isthmocoele. Conclusion: Cervico-isthmic pregnancy is rare. His treatment is not codified. Drug management was successful.
\end{abstract}

\section{Keywords}

Cervico-Isthmic Pregnancy, Medical Treatment, Methotrexate

\section{Introduction}

Cervical-isthmic pregnancy (CIP) is a rare form of ectopic pregnancy. It often occurs on caesarean scars, preferably multiple scars [1] [2]. Due to the increasing number of caesarean deliveries, the incidence of CIP varies from 1/1000 to $1 / 95,000$ [3]. It is particularly serious because of its hemorrhagic complication 
which can compromise the patient's vital prognosis. Contrary to cervical pregnancy which rarely exceeds the $20^{\text {th }}$ week, CIP on caesarean scar can evolve to term, threatening the patient any moment with uterine rupture, cataclysmic hemorrhage due to the low insertion of the placenta on a richly vascularized inferior segment [4]. Cervical-isthmic pregnancy must therefore be sought out and diagnosed in full, especially in the case of a history of multiple caesarean sections, in order to establish proper management and thus avoid complications. When diagnosed early, its management is very controversial about its evacuation technique.

Through this observation, we report our experience in the management of a CIP.

\section{Case Presentation}

This is 35-year-old women who consulted in the gynecological emergency room for pelvic pain and delayed menstruation. She is G2P2, with two cesarean deliveries. Clinical examination notes diffuse pelvic pain and minimal bleeding. The biological assessment noted a bHCG rate at 20,000 IU/L and a progesterone rate at $25 \mathrm{ug} / \mathrm{L}$. The vaginal ultrasound found a retroverted uterus with a regular gestational sac containing a living embryo of 6SA, on the caesarean scar (Figure 1: gestational sac implanted on the cesarean scar, just under the perimetrium, Figure 2: embryo with heart activity, Figure 3: gestational sac with yolk sac on cervico-isthmic portion). The uterine fundus was empty, the ovaries normal. After explaining the severity of this pregnancy and the risk of uterine rupture, if the pregnancy continues, the patient decides to terminate the pregnancy, it was decided in agreement with the patient, a therapeutic abortion. Different evacuation options were explained to the patient who opted for medical evacuation. Thus, $600 \mathrm{mg}$ of mifepristone was oral administered, followed by oral administration of $400 \mu \mathrm{g}$ of Misoprostol and a single dose of Methotrexate $1 \mathrm{mg} / \mathrm{kg}$ intramuscularly, 48 hours after mifepristone was given. Clinical and biological monitoring with bHCG control was then instituted on the 4th and 7th day, then every week until bHCG was negative. Evolution was favorable with negativation

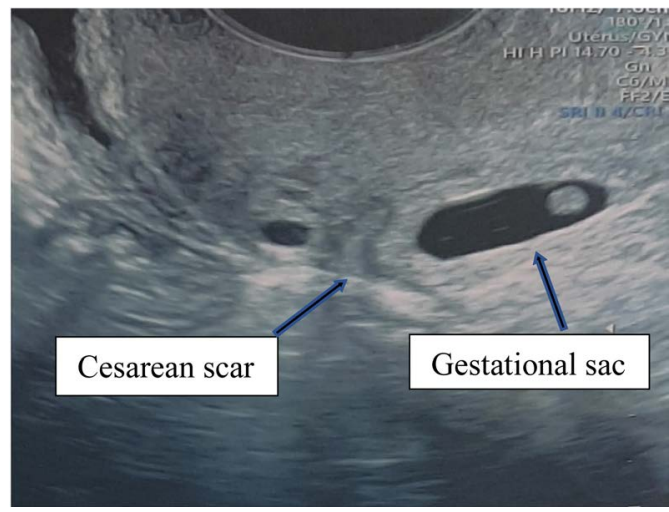

Figure 1. Gestational sac implanted on the cesarean scar. 


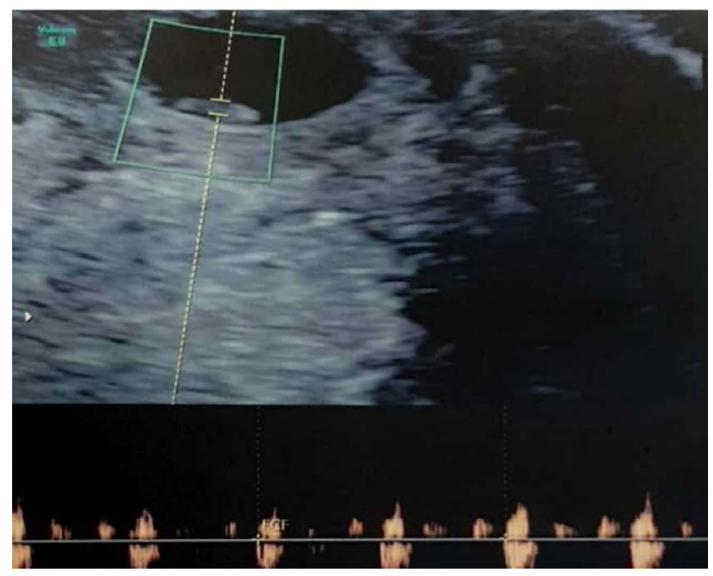

Figure 2. Embryo with heart activity.

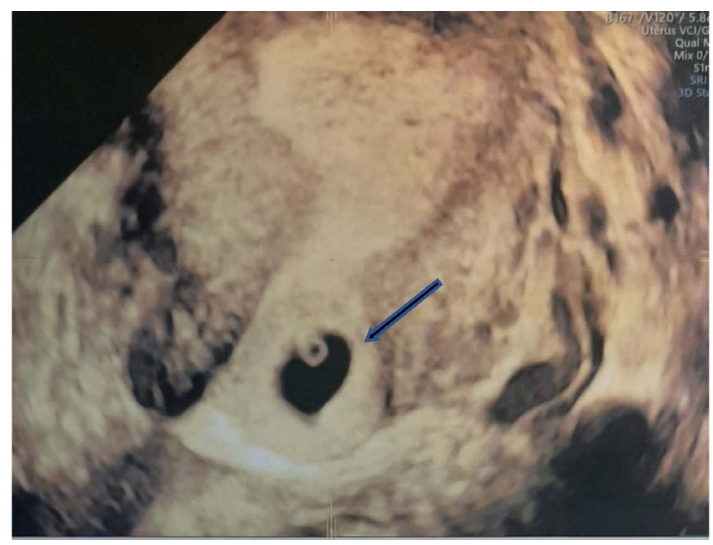

Figure 3. Coronal view, the arrow shows a gestational sac with yolk sac.

of bHCGs one month later. Histological examination of the product of conception confirmed the diagnosis. An ultrasound performed 2 months later noted the uterine vacuity and an isthmocele where the pregnancy had implanted (Figure 4).

\section{Discussion}

\subsection{Frequency}

The exact frequency of CIP is not well known. It varies from 1/1000 to $1 / 95,000$ with an estimated average of $1 / 20,000$ [3]. They represent approximately $1 \%$ of all ectopic pregnancies [5] [6] [7]. Considering pregnancy due to cesarean scar, its incidence would be increasing due to the increasing number of cesarean deliveries. It represents around $6.1 \%$ of ectopic pregnancies [8].

\subsection{Pathophysiology}

The pathophysiology of CIP is not well known. It would be the consequence of an implantation of the gestational sac in the lower part of the uterus with a secondary extension in the isthmus, or a cervical location with extension to the 


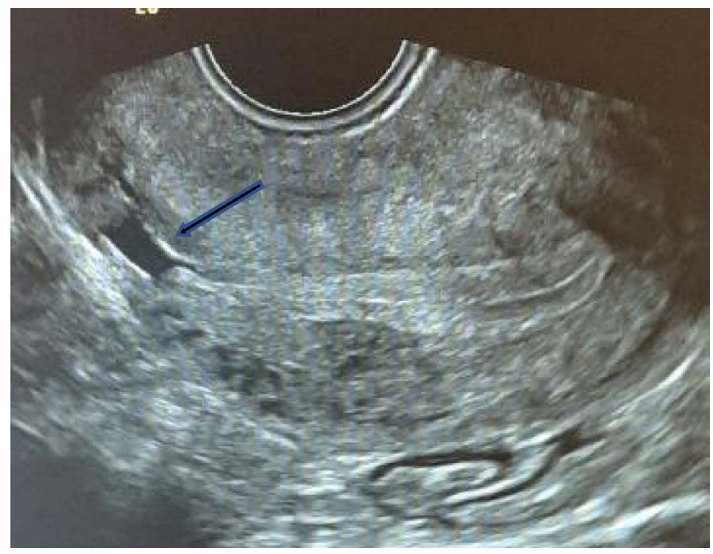

Figure 4. The arrow shows an isthmocele.

uterine isthmus [9] [10]. More recently, it has been described as a complication of voluntary terminations of pregnancy by secondary cervical reimplantation [11]. The factors favoring these pregnancies are related to an alteration of the endometrium to accommodate the fertilized egg, or of the cervical canal during cervical dilation or curettage maneuvers. We thus find a history of cesarean section, endo-uterine maneuvers, uterine malformations, endometrial atrophy, endometritis or in vitro fertilization (IVF) [3] [12]. Studies have reported an increased incidence of cervical pregnancies in women who achieved their pregnancies through in vitro fertilization [13] [14].

\subsection{Diagnostic}

The clinical diagnosis of CIP remains difficult. The clinical signs are inconsistent, ranging from the complete absence of functional signs to the classic triad of ectopic pregnancy including delayed menstruation, pelvic pain and bleeding. Most often, it manifests itself by isolated metrorrhagia as our case report. At first, except for uterine bleeding, the clinical examination is poor as our case report or an isthmus mildly distended and softened at the digital examination [15]. The diagnosis is done by vaginal ultrasound which shows the gestational sac with its trophoblastic in the cervico-isthmic portion and an empty uterine fundus. The absence of the "sliding sign" and the vascularization of the gestational sac on Doppler confirms cervico-isthmic implantation of the pregnancy and eliminates an ongoing miscarriage.

\subsection{Treatment}

The management of cervical pregnancies is not yet well codified and varies from team. This treatment ranges from therapeutic abstention to radical hysterectomy and hemostasis. Indeed, the literature has described an evolution of cervico-isthmic pregnancies until fetal viability, or even term [9] [16]. On the other hand, the cervical portion being well vascularized and lacking the muscular competence required to ensure the development of a pregnancy, the course will lead to spontaneous abortions very hemorrhagic by lack of "living" ligatures. 
The management of IPC depends on clinical signs, gestational age, viability of the pregnancy, extent of isthmus neovascularization and patient preference [17]. Medical treatment consists of intramuscular injection of $1 \mathrm{mg} / \mathrm{kg}$ of Methotrexate. Authors also describe the combination of Mifepristone and misoprostol with Methotrexate to facilitate the expulsion of the product of conception, or the ultrasound guided injection of KCL in pregnancy if cardiac activity is present [7] [18]. For our case report, we used the protocol combining Mifepristone and misoprostol followed by the administration of a single dose of Methotrexate 1 $\mathrm{mg} / \mathrm{kg}$. Cure was obtained after one month by the negativation of the plasma level of HCG. We had no bleeding complications in our case. A similar case was reported at 8 weeks by Tinelli A. et al. [19] where despite the administration of methotrexate, a laparoscopy was performed for rupture of CIP at 8 weeks. Another case was reported at 7 weeks where a hysteroscopic resection of the pregnancy was performed after failure of systemic administration and direct injection with hysteroscopic view of MTX [15]. Because of isthmus neovascularization and hemorrhagic risk, many teams propose complementary interventions to any pregnancy evacuation procedure, such as uterine tamponade, ligation of the hypogastric arteries, embolization of the uterine arteries, or hemostasis hysterectomy [5] [7] [20].

\section{Conclusion}

Cervico-isthmic pregnancy is rare, its pathophysiology is not well known but it usually occurs on a caesarean scar. The diagnosis is made in the 1st trimester by vaginal ultrasound. The main danger is its haemorrhagic complication which can be cataclysmic. Its management is not unanimous. Depending on the team, it can be treated with medication or surgery.

\section{Ethical Considerations}

We have obtained patient's consent, and reassured that information could only be used in a science-related purpose.

\section{Conflicts of Interest}

The authors declare no conflicts of interest regarding the publication of this paper.

\section{References}

[1] Kung, F.T., Huang, T.L., Chen, C.W. and Cheng, Y.F. (2006) Image in Reproductive Medicine. Cesarean Scar Ectopic Pregnancy. Fertility and Sterility, 85, 1508-1509. https://doi.org/10.1016/j.fertnstert.2005.12.016

[2] Timor-Tritsch, I.E. and Monteagudo, A. (2012) Unforeseen Consequences of the Increasing Rate of Cesarean Deliveries: Early Placenta Accreta and Cesarean Scar Pregnancy. A Review. American Journal of Obstetrics \& Gynecology, 207, 14-29. https://doi.org/10.1016/j.ajog.2012.03.007

[3] Riethmuller, D., Courtois, L., Maillet, R. and Schaal, J.P. (2003) Ectopic Pregnancy 
Management: Cervical and Abdominal Pregnancies. Journal de Gynecologie, $O b-$ stetrique et Biologie de la Reproduction, 32, S101-S108.

[4] Jurkovic, D., Hillaby, K., Woelfer, B., Lawrence, A., Salim, R. and Elson, C.J. (2003) First Trimester Diagnosis and Management of Pregnancies Implanted into the Lower Uterine Segment Cesarean Section Scar. Ultrasound in Obstetrics \& Gynecology, 21, 220-227. https://doi.org/10.1002/uog.56

[5] Verma, U. and Goharkhay, N. (2009) Conservative Management of Cervical Ectopic Pregnancy. Fertility and Sterility, 9, 671-674. https://doi.org/10.1016/j.fertnstert.2007.12.054

[6] Verma, U., English, D. and Brookfield, K. (2011) Conservative Management of Non Tubal Ectopic Pregnancies. Fertility and Sterility, 96, 1391-1395.

https://doi.org/10.1016/j.fertnstert.2011.09.021

[7] Chetty, M. and Elson, J. (2009) Treating Non-Tubal Ectopic Pregnancy. Best Practice \& Research: Clinical Obstetrics \& Gynaecology, 23, 529-538. https://doi.org/10.1016/j.bpobgyn.2008.12.011

[8] Shikha, J., Neha, J. and Swati C. (2016) Embryo Implantation in the Region of a Previous Caesarean Section Scar and Scar Dehiscence in Second Trimester: A Rare Case Report. The Journal of Obstetrics and Gynecology of India, 66, S613-S616. https://doi.org/10.1007/s13224-016-0874-2

[9] David, M.P., Bergman, A. and Delighdish, L. (1980) Cervico-Isthmic Pregnancy Carried to Term. Obstetrics \& Gynecology, 56, 247-252.

[10] Oyelese, Y., Elliott, T.B., Asomani, N., Hamm, R., Napoli, L. and Lewis, K.M. (2003) Sonography and Magnetic Resonance Imaging in the Diagnosis of Cervico-Isthmic Pregnancy. Journal of Ultrasound in Medicine, 22, 981-983. https://doi.org/10.7863/jum.2003.22.9.981

[11] Pizzoferrato, A.C., Legendre, G., Demaria, F. and Benifla, J.L. (2012) Cervical Pregnancy: A Rare Case of Reimplantation after Abortion. A Case Report. Journal de Gynécologie Obstétrique et Biologie de la Reproduction, 41, 587-590. https://doi.org/10.1016/j.jgyn.2012.07.002

[12] Hung, T., Jeng, C.J., Yang, Y.C., Wang, K.G. and Lan, C.C. (1996) Treatment of Cervical Pregnancy with Metothrexate. International Journal of Gynecology \& $\mathrm{Ob}$ stetrics, 53, 243-247. https://doi.org/10.1016/0020-7292(96)02653-7

[13] Yan, O., Xihong, L., Yan, Y., Fei, G., Ge, L. and Guangxiu, L. (2015) First-Trimester Diagnosis and Management of Cesarean Scar Pregnancies after in Vitro Fertilization-Embryo Transfer: A Retrospective Clinical Analysis of 12 Cases. Reproductive Biology and Endocrinology, 13, 126. https://doi.org/10.1186/s12958-015-0120-2

[14] Hulvert, J., Mardesic, T., Voboril, J. and Muller, P. (1996) Heterotopic Pregnancy and Its Occurrence in Assisted Reproduction. Czech Gynaecology, 64, 299-301.

[15] Sardo, A.D.S., Alviggi, C., Zizolfi B., Spinelli M., De Rosa, P., De Placido, G. and Nappi, C. (2013) Cervico-Isthmic Pregnancy Successfully Treated with Bipolar Resection Following Methotrexate Administration: Case Report and Literature Review. Reproductive Bio Medicine Online, 26, 99-103. https://doi.org/10.1016/j.rbmo.2012.10.005

[16] Itakura, A., Okamura, M., Ohta, T. and Mizutani, S. (2003) Conservative Treatment of a Second Trimester Cervicoisthmic Pregnancy Diagnosed by Magnetic Resonance Imaging. Obstetrics \& Gynecology, 101, 1149-1151. https://doi.org/10.1097/00006250-200305001-00038

[17] Herman, A., Weinraub, Z., Avrech, O., Maymon, R., Ron-El, R. and Bukovsky, Y. 
(1995) Follow-Up and Outcome of Isthmic Pregnancy Located in Previous Cesarean Section Scar. British Journal of Obstetrics and Gynaecology, 102, 839-841. https://doi.org/10.1111/j.1471-0528.1995.tb10855.x

[18] Kung, F.T. and Chang, S.Y. (1999) Efficacy of Methotrexate Treatment in Viable and Nonviable Cervical Pregnancies. American Journal of Obstetrics \& Gynecology, 181, 1438-1444. https://doi.org/10.1016/S0002-9378(99)70389-3

[19] Tinelli, A., Tinelli, R. and Malvasi, A. (2009) Laparoscopic Management of Cervico-Isthmic Pregnancy: A Proposal Method. Fertility and Sterility, 92, 2. https://doi.org/10.1016/j.fertnstert.2009.05.020

[20] Fylstra, D.L. and Coffey, M.D. (2001) Treatment of Cervical Pregnancy with Cerclage, Curettage and Balloon Tamponade. A Report of Three Cases. Journal of Reproductive Medicine, 46, 71-74. 